\title{
Evaluation of Surface Slope Irregularity in Linear Parabolic Solar Collectors
}

\author{
F. Francini, D. Fontani, P. Sansoni, L. Mercatelli, D. Jafrancesco, and E. Sani \\ National Institute of Optics (INO), CNR, Largo E. Fermi, 6, 50125 Firenze, Italy \\ Correspondence should be addressed to F. Francini, franco.francini@ino.it
}

Received 21 June 2011; Revised 11 October 2011; Accepted 17 October 2011

Academic Editor: Xie Quan

Copyright () 2012 F. Francini et al. This is an open access article distributed under the Creative Commons Attribution License, which permits unrestricted use, distribution, and reproduction in any medium, provided the original work is properly cited.

The paper describes a methodology, very simple in its application, for measuring surface irregularities of linear parabolic collectors. This technique was principally developed to be applied in cases where it is difficult to use cumbersome instruments and to facilitate logistic management. The instruments to be employed are a digital camera and a grating. If the reflector surface is defective, the image of the grating, reflected on the solar collector, appears distorted. Analyzing the reflected image, we can obtain the local slope of the defective surface. These profilometric tests are useful to identify and monitor the mirror portions under mechanical stress and to estimate the losses caused by the light rays deflected outside the absorber.

\section{Introduction}

The distribution of solar irradiance values on the receiver tubes, used in linear parabolic collectors, depends on the geometric characteristics of the reflector. In particular, the uniformity of irradiation over the entire length of the tube depends on the regularity of the reflector profile along the longitudinal axis and on the presence or absence of localized defects that can direct sunlight to a region of space outside the receiving tube.

The collection efficiency of a solar trough system is affected by several types of mirror surface defects: profile deformation, structural torsion, and local irregularities. In particular the local faults can be of microscale (microns in high) or of macroscale (millimeters in high). The surface microirregularities are identifiable with the surface roughness, which only introduces efficiency losses due to the diffused light. This paper discusses a technique to examine macroscopic scale defects in the reflector profile, to quantify their size and to assess their effect on system performance.

The methods used to detect profile defects are mainly based on geometric optics techniques; the procedure is to identify the direction of a light beam hitting a specific point of the reflector, and to verify that the reflected beam is deflected according to the nominal value of the surface local slope.
The methods described in literature [1] are very timeconsuming because they refer to specific tests obtained by scanning the surface under examination. Obviously higher resolutions require longer times to complete the measurement.

In alternative, we could employ techniques "image processing" that allow analyzing, in a reasonably short time, the entire surface of the reflector. The methods involving these techniques mostly perform a distant detection of the image of the absorber tube, which is formed by the reflector. This optical arrangement is equivalent to observe an object (the tube), through an optical system (the reflector), able to zoom the object. If the viewing distance is much longer than the focal length of the reflector, the visible faraway image covers the whole reflector, and the observer can see an image of uniform color. Papers [2-4] describe cases where profile irregularities deteriorate the image in the reflector defective points, which appear as areas of different intensity. Other procedures refer to the analysis of a calibrated mask whose image is reproduced by the concentrator [5-8]. A method using a colored pattern, projected on a screen through the concentrator, is explained in reference [9]. An alternative approach exploits photogrammetric techniques [10]. Some recent researches include like fringe reflection method [11-13] and a 3D optical profilometry technique [14]. 
Detection procedure, measurement configuration, and optoelectronic devices of our proposed methodology were especially developed for applications where it is difficult to install support structures and to use sophisticated instrumentation. The procedure consists in covering the absorbing tube with a grid, printed on a paper sheet, and to analyze from a known distance its image, reflected by the linear solar mirror. Figure 1 presents an image of the reference grating reflected on the concentrator. Image of the reference grating reflected on the concentrator. It is very convenient to place the grating on the tube but is not strictly necessary; the grating can be placed at any position in front of the reflector.

\section{Experimental Setup}

Figure 2 shows a scheme of the measuring experimental system, evidencing angles and distances to be considered between concentrator and digital photographic camera, used to capture an image of the grating. Since the surface to be examined is reflective, the image of the grating is virtual, and it is formed beyond the surface, as indicated in Figure 2.

All defects on the reflector surface are represented as local variations in slope. Assuming that the metal pipe approaches a regular cylinder, the defects appearing on the image are due to a defective reflector surface. In other words, a perfect reflector produces an undistorted image of the grating whose size depends on the magnification factor $M$ of the optical system. The dimension of the observed image increases with the distance of the observer from the mirror. If we suppose to cover the absorbing tube with a grating of step $p$, this will appear distorted if the defect will be larger than $p \times M$. Consequently to detect small defects, using the same step $p$, we had to take pictures of the tube at a reasonably short distance. As shown in Figure 1, the image of the tube appears inside of the reflector. Therefore, in order to explore the entire reflector surface we must change the high of the photographic camera. The complete detection can be done moving the digital camera (DgC) from top to bottom, without changing the position of the grid. Referring to Figure 2, given a generic point $\mathrm{P}$ on the reflector, the corresponding angle $\theta$ is given, with good approximation, by the following relations:

$$
\theta=\tan ^{-1} \frac{y}{f-z} \quad z=c y^{2} \quad c=\frac{1}{R},
$$

where $f$ represents the parabola focal distance, $R$ is the radius of curvature, $C$ is the reflector curvature (referred to the ideal parabola), and $y, z$, and $\theta$ are defined in Figure 2 . The $y$ values are determined by the position of the rays connecting image and photographic camera. These values are derived from the grating image by detecting the positions of its lines. The real dimensions of the image are obtained referring to a calibrated marker placed on the reflector surface or taking as reference an object of known size.

\section{Defect Detection Using Structured Light Techniques}

A 3D recontruction of the examined reflecting surface can be obtained elaborating the detected image. Within the grating

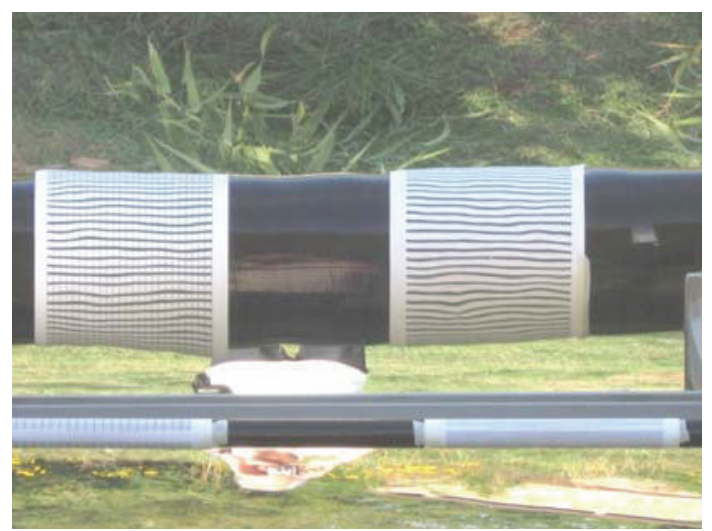

FIGURE 1: Image of the reference grating reflected on the concentrator.

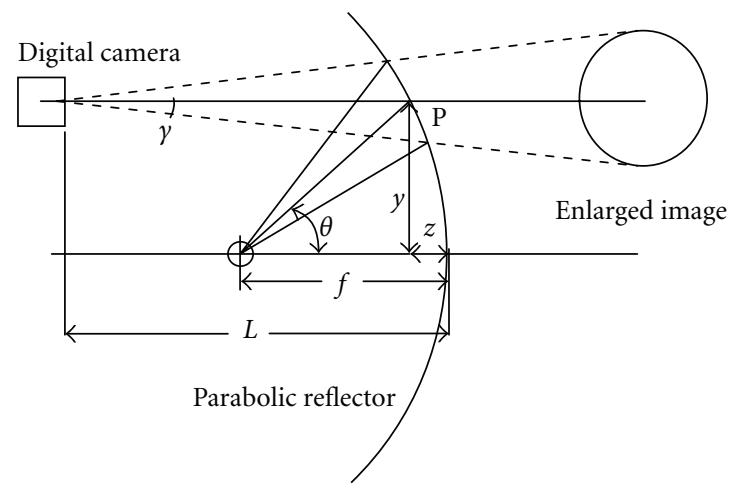

FIGURE 2: Scheme of the measuring experimental system.

image reflected by the mirror surface, the deformed lines indicate slope variations. These defects can be extracted from the image using structured light techniques [15-17] adapted to our specific case. The adaptation consists in applying structured light procedures on reflectors, while they are typically used on diffusing surfaces.

The theory indicates that we can measure the object altitude $z$ projecting a bright spot on the surface under study with simple geometric relationships. Figure 3 summarizes the working principle of structured light techniques.

This technique needs the registration of two images: the first belongs to the reference plane $\xi$ without the object and the second to the measured object located on the plane $\xi$.

Consequently, the surface is lighted the first time in the point $P_{1}$ and the second time in the point $P_{2}$. The comparison of the two images evidence the displacement from $P_{1}$ to $\mathrm{P}_{2}$ as detected spot shift $p^{\prime}$, which is connected to the local height $z$ by the following equations:

$$
p=\frac{p^{\prime}}{M}, \quad \theta_{0}=\arctan \frac{b}{h}, \quad z=\frac{p}{\tan \theta_{0}},
$$

where $h, b, p$, and $\theta_{0}$ are defined in Figure 3 , and $M$ represents the magnification of the optical system.

The structured light techniques need to be modified to monitor reflecting objects. Considering a diffusing surface, the detected spot shift is related only to the altitude of 


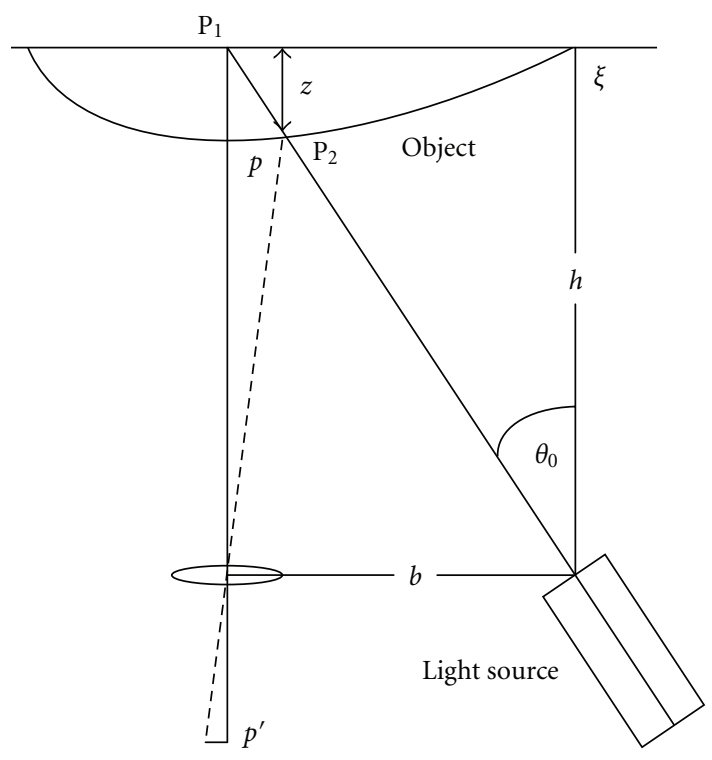

Figure 3: The principle of structured light techniques.

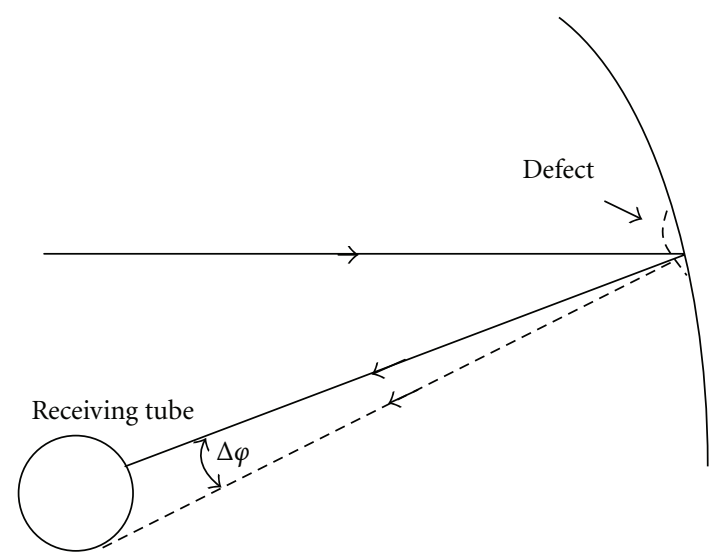

FIgURE 4: Rays deviation in the direction of the receiver tube.

the measurement point. But in case of a mirror, the detected spot shift depends on the slope of the surface. The foremost parameter to be measured is the slope of the local defect, because it combines high and extension of the local defect. The defect slope allows to immediately determining if the impinging light is reflected outside the receiving tube; if the deviation is higher than $\Delta \varphi$, the ray does not hit the tube, as shown in Figure 4. Figure 4 presents the deviation of rays in the direction of the receiver tube.

The slope differences due to surface irregularities can be identified referring to Figures 5 and 6 . Figure 5 represents a detail of the reflector, evidencing the local slope $\varphi$ of the surface in the observation point. $\gamma$ represents the angle of the ray that goes from the reflector to the digital camera lens.

Considering the following relations:

$$
\begin{gathered}
\alpha+\beta+\varphi=90, \\
\beta=90-\theta, \\
2 \alpha=180-\beta-(90-\gamma),
\end{gathered}
$$

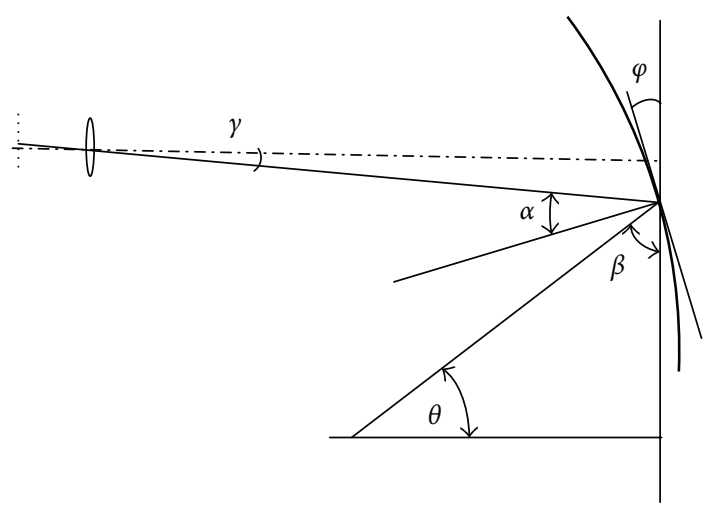

FIGURE 5: Detail of the reflector evidencing the local slope $\varphi$ of the surface in the observation point.

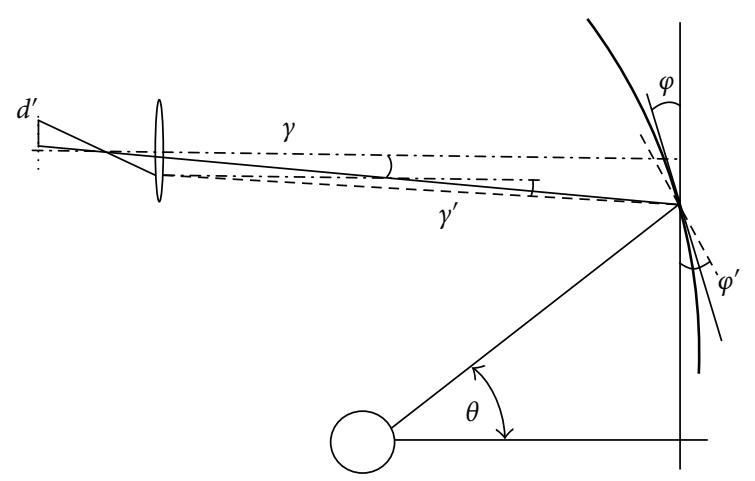

Figure 6: Detail of the reflector, schematically introducing a slope defect.

we can obtain the relationship between the local inclination $\varphi$ and the angle $\gamma$

$$
\varphi=\frac{\theta-\gamma}{2} .
$$

Figure 6 shows a detail of the reflector, schematically introducing a slope defect $\left(\varphi^{\prime}\right)$. The optical rays, arriving on the photographic camera, are marked with a dashed line if they come from the defect and with a solid line if they come from the ideal surface.

The angle $\varphi^{\prime}$ indicates the local slope of the surface in presence of the defect, and $d^{\prime}$ represents the difference between the position of the reference line and that effectively measured in case of defect presence. The values of $d^{\prime}$ are obtained from the image taken with the camera DgC considering a virtual reference grating projected on the ideal parabolic reflector profile. Having obtained the value of $d^{\prime}$ by the recorded images, we can calculate the difference $\Delta \gamma$ between the two angles $\gamma$ and $\gamma^{\prime}$, knowing the distance $L_{p}$ between the measuring point and the photographic camera.

$\Delta y$ is obtained through the following relationship:

$$
d^{\prime}=M \cdot L_{p} \cdot \tan \Delta \gamma, \quad \Delta y=\operatorname{atn} \frac{d^{\prime}}{M \cdot L_{p}},
$$

where $M$ is the magnification of the optical system $(M$ is $<1)$. 


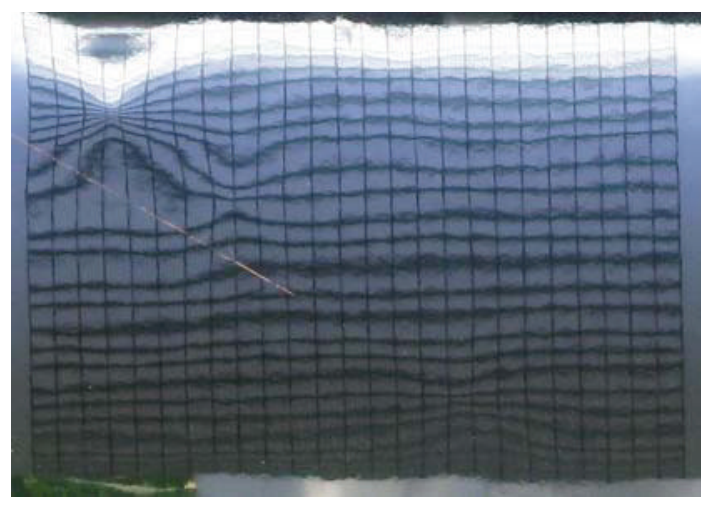

Figure 7: Image of the grating distorted by a defect.

Consequently the difference of slope $\Delta \varphi$ can be obtained using (4) as follows:

$$
\begin{gathered}
\Delta \varphi=\varphi-\varphi^{\prime}=\frac{\gamma-\gamma^{\prime}}{2}, \\
\Delta \varphi=\frac{\Delta \gamma}{2} .
\end{gathered}
$$

\section{Experimental Results}

Figure 7 shows a portion of the concentrator that reflects the image of the grating. The image was taken in correspondence of an air bubble under the reflective surface, formed during the operation of gluing a reflective aluminum sheet on the concentrator structure. The experimental results were obtained for a linear parabolic concentrator with a focal length of $800 \mathrm{~mm}$ and a $1600 \mathrm{~mm}$ entrance aperture. The photographic camera is located at a distance of $2 \mathrm{~m}$ from the parabola vertex, and it is placed at a height $y$ of $400 \mathrm{~mm}$. The measurement configuration is shown in Figure 2. The grating is superimposed on the receiving tube of the parabolic solar trough.

Figure 8 represents an elaboration of Figure 7 aimed to highlight the spatial information (two-level binary image). The horizontal and vertical lines visible in the image in Figure 7 were derived from a bidimensional grid placed on the receiver tube. This grid allows a bidimensional analysis of the image, which is carried out separately for rows and columns. The horizontal lines are spaced of $10 \mathrm{~mm}$, while the vertical lines are spaced of $2.5 \mathrm{~mm}$.

Concerning the grid application, having to be placed on the tube, the grid obviously assumes its same curvature. Consequently the columns are not deformed, while the rows present variable spacing, which decreases from the tube image center towards borders.

Figure 9 represents an example of a color map reconstruction of the slope differences $\Delta \varphi$ between the ideal surface of the reflector and the defective one, considering the horizontal lines. Figure 10 has been obtained from the image of Figure 7 considering only the vertical lines. Examination of the vertical lines allows us to calculate the slope of the defective surface in the longitudinal direction of the reflector.

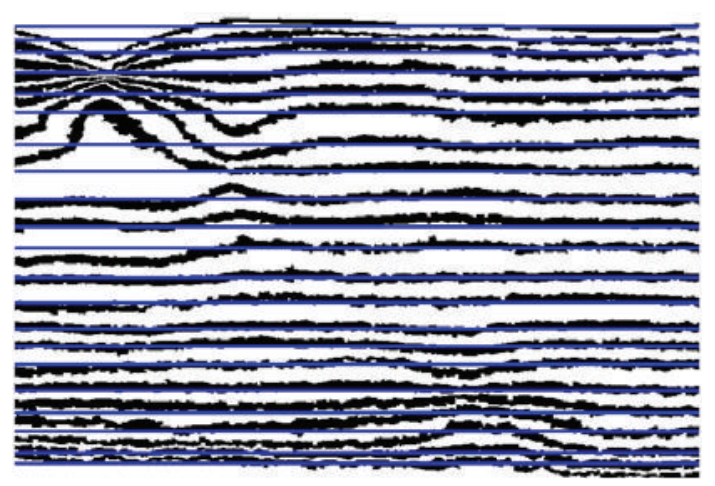

FIGURE 8: Elaboration of Figure 7 aimed to highlight the spatial information (two-level binary image) of the horizontal lines. In the figure have been superimposed the horizontal reference lines.

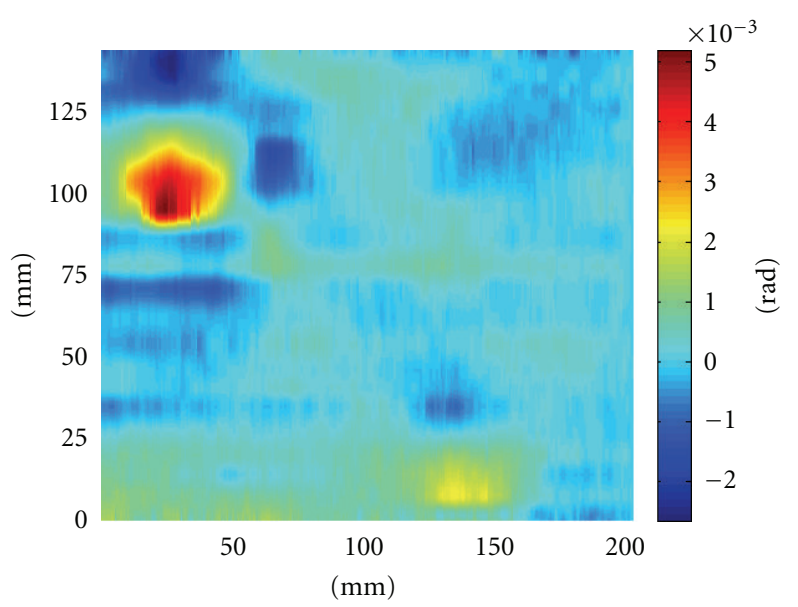

FIGURE 9: Color map reconstruction of slope differences $\Delta \varphi(\mathrm{rad})$ considering horizontal lines.

The calculation methodology is identical to that used in Figure 8, reconsidering the direction of the angles $\varphi$ and $\gamma$.

The information obtained from the grating image can be elaborated as previously described, obtaining the 2D map of the slopes, as shown in Figure 11.

In alternative the image information supplies position and number of irregularities, but also the spread of the light spot on the absorber caused by the defect, in addition to the natural spread due to solar divergence. This investigation is useful especially in the process of placement and adjustment of the solar plant. In particular to check that the means used to fix the mirrors on the frame of the concentrator do not interfere with mirrors nominal profile, an example is shown in Figure 7: a mirror deformed by a gluing operation.

Basically the 2D slope reconstruction of the real reflector represents a qualitative assessment on the reflector correspondence to the nominal profile. It also provides information about the collection efficiency performance of a system. For a more detailed analysis, it will be possible to join together the nominal surface of the reflector with the measured one, to evidence defective zones. 


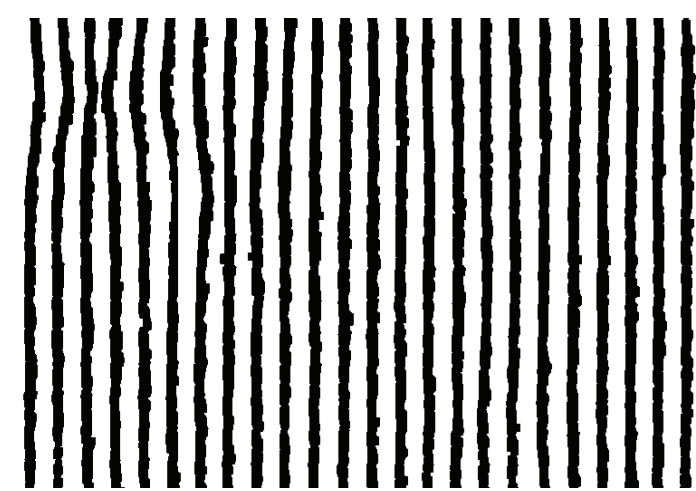

Figure 10: Elaboration of Figure 7 aimed to highlight the spatial information (two-level binary image) of the vertical lines.

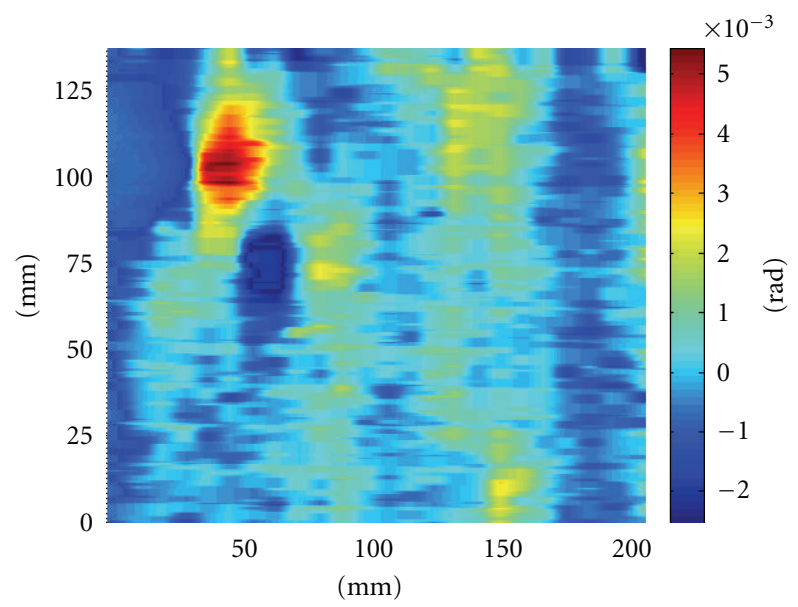

Figure 11: Color map reconstruction of slope differences $\Delta \varphi$ (rad) considering vertical lines.

\section{Conclusions}

The described technique is addressed to field tests and measurements on operating solar plants, avoiding the use of complex mechanical structures and sophisticated equipments. Furthermore, the detection system was studied to be lightweight, easily transportable and with an extreme facility of alignment. The proposed surface quality test can be performed with minimal instrumentation; only a digital camera and a drawn grid are needed. The optoelectronic device is commonly used and inexpensive; the detection configuration is easily reproducible, and the measurement procedure is very simple.

The immediate and simplified result consists in examining the grating image, which can already assesses the surface quality. The deformations visible on the grading image can classify the reflecting surface; moreover, they localize the imperfections and give information on defect magnitude (on high and extent). Hence, this technique represents a fast and efficient method to detect the surface irregularities of a reflective parabolic concentrator.

Beyond the use for this visual inspection on solar plants, the method is able to estimate a numerical value for the slope.
From the deformed grating image, we can obtain a 2D map of mirror slope variations. This reconstruction procedure is the most complex and time-consuming part of the method. The elaboration methodology was deeply discussed and analyzed, presenting examples of results as deformed grating image and surface slope reconstruction.

\section{Acknowledgments}

The work was developed within the Solar Assisted Cooling Toscana. (S.A.L.TO.) research integrated Project POR Ob. 3 Toscana 2000/2006 Misura D4, partially financed by the REGIONE TOSCANA - settore Promozione e sostegno della Ricerca. Thanks are due to CREAR and Department of Engineering of the Florence University for the realization of solar cooling systems, in collaboration with the industrial partners, FAIT group, and CEVIT.

\section{References}

[1] A. Maccari and M. Montecchi, "An optical profilometer for the characterisation of parabolic trough solar concentrators," Solar Energy, vol. 81, no. 2, pp. 185-194, 2007.

[2] S. Ulmer, B. Heinz, K. Pottler, and E. Lüpfert, "Slope error measurements of parabolic troughs using the reflected image of the absorber tube," Journal of Solar Energy Engineering, vol. 131, no. 1, pp. 0110141-0110145, 2009.

[3] D. Krüger, Y. Pandian, K. Hennecke, and M. Schmitz, "Parabolic trough collector testing in the frame of the REACt project," Desalination, vol. 220, no. 1-3, pp. 612-618, 2008.

[4] R. B. Diver and T. A. Moss, "Practical field alignment of parabolic trough solar concentrators," Journal of Solar Energy Engineering, vol. 129, no. 2, pp. 153-159, 2007.

[5] M. Avendaño-Alejo, V. I. Moreno-Oliva, M. Campos-García, and R. Díaz-Uribe, "Quantitative evaluation of an off-axis parabolic mirror by using a tilted null screen," Applied Optics, vol. 48, no. 5, pp. 1008-1015, 2009.

[6] M. Campos-García, R. Díaz-Uribe, and F. Granados-Agustín, "Testing fast aspheric convex surfaces with a linear array of sources," Applied Optics, vol. 43, no. 34, pp. 6255-6264, 2004.

[7] V. I. Moreno-Oliva, C. Garcia, F. Granados-Augustin, M. J. Arjona-Perez, D. Uribe, and A. Alejo, "Optical testing of a parabolic trough solar collector by null screen with stiching," in Optical MetrologyII, vol. 7390 of Proceedings of SPIE in Modelling Aspects, October 2009.

[8] T. Wendelin, "Parabolic trough VSHOT optical characterization in 2005-2006," in Proceedings of the Parabolic Trough Technology Workshop, NREL/PR-550-40024, Incline Village, Nev, USA, February 2006.

[9] D. Fontani, F. Francini, L. Mercatelli, D. Jafrancesco, and P. Sansoni, "Colour coded methodology for deformable mirrors," Issue of Optical Engineering, vol. 45, no. 8, p. 080508 , 2006, nostro lavoro O.E.

[10] K. Pottler and E. Lupfert, "Photogrammetry: a powerfullTool for geometrical analysis of solar concentrators and their componentents," Journal of Solar Energy Engineering, vol. 127, pp. 94-101, 2005.

[11] C. Andraka, K Chavez, N. Finch, C. Ghanbari, E. Sproul, and J. Yellowhair, "Sofast facet characterization for heliostats: extensions and difficulties," in Proceedings of the SolarPaces Conference, Book of Abstracts, p. 64, Granada, Spain, September 2011. 
[12] A. Heimsath, G. Bern, and P. Nits, "Shape accuracy of solar mirrors-comparison of two methods using fringe reflection technique," in Proceedings of the SolarPaces Conference, Book of Abstracts, p. 76, Granada, Spain, September 2011.

[13] A. Lehmann, "Rapid heliostat surface measurement using reconstruction from camera images," in Proceedings of the SolarPaces Conference, Book of Abstracts, p. 70, Granada, Spain, September 2011.

[14] M. Montecchi, A. Benedetti, and G. Cara, "Fast 3D opticalprofilometer for shape-accuracy control of parabolic-trough facets," in Proceedings of the SolarPaces Conference, Book of Abstracts, p. 298, Granada, Spain, September 2011.

[15] Kjell J. Gasvik, Optical Metrology, John Wiley \& Sons, Chichester, UK, 1995.

[16] O. Kafri and I. Glatt, The Physics of Moire Metrology, Wiley series in pure and applied optics, John Wiley \& Sons, New York, NY, USA, 1990.

[17] P. K. Rastogi and D. Inaudi, Trend in Optical Nondescructive Testing and Inspection, Elsevier, Oxford, UK, 2000. 


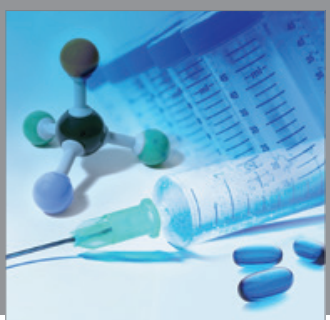

International Journal of

Medicinal Chemistry

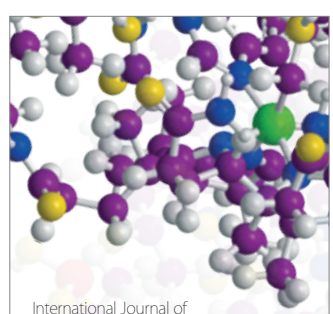

Carbohydrate Chemistry

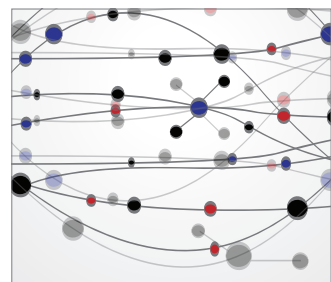

The Scientific World Journal
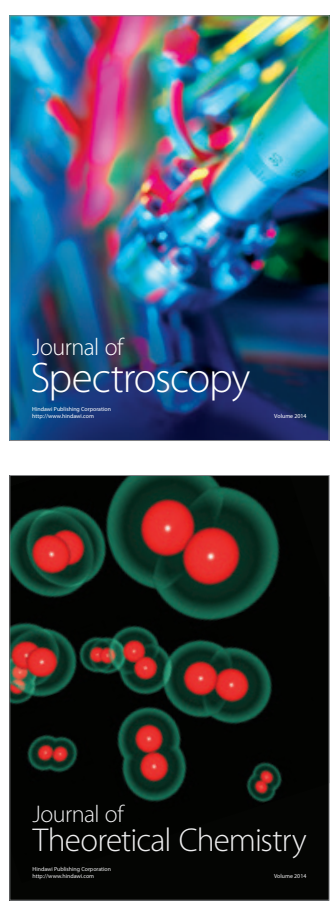
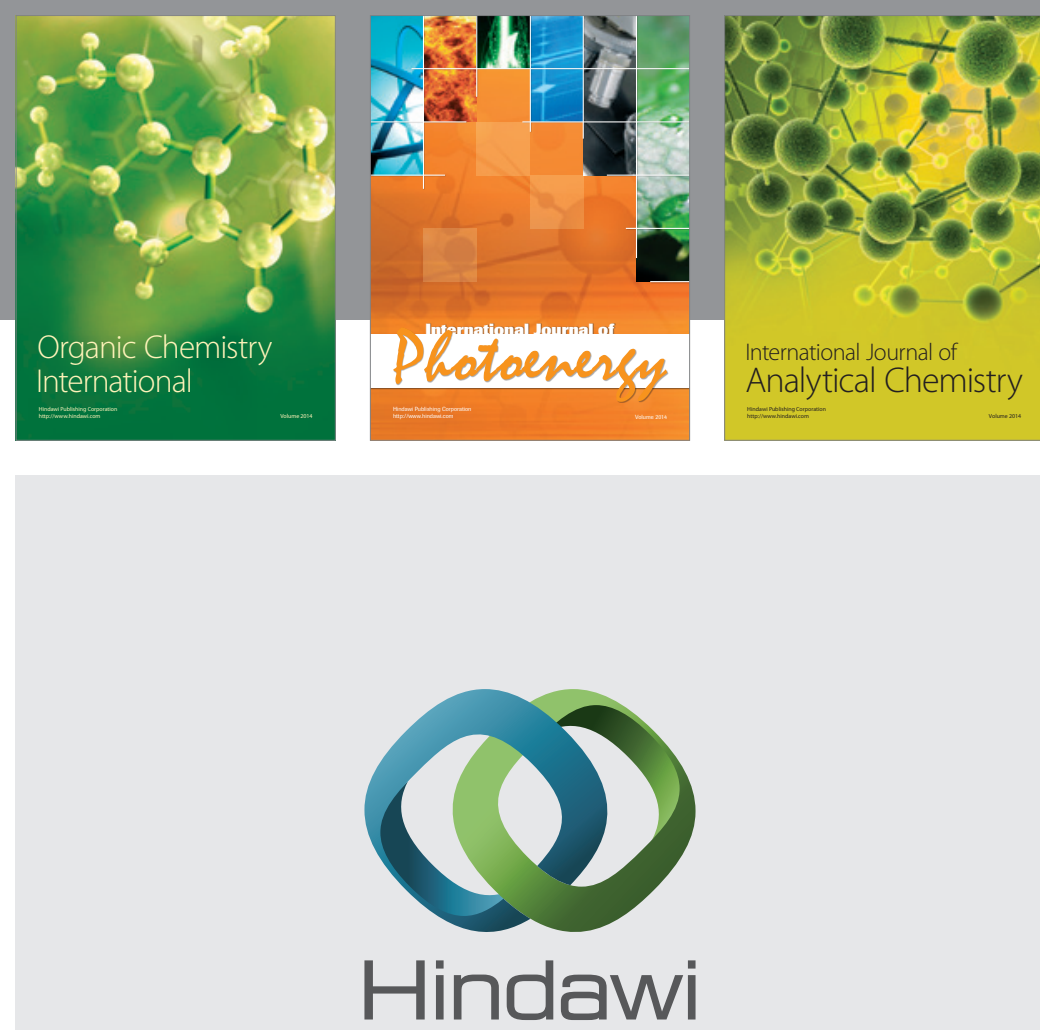

Submit your manuscripts at

http://www.hindawi.com
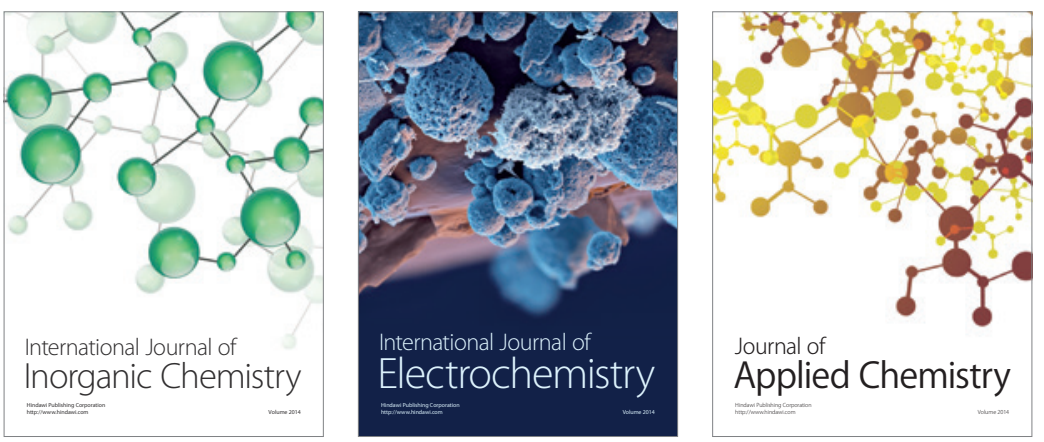

Journal of

Applied Chemistry
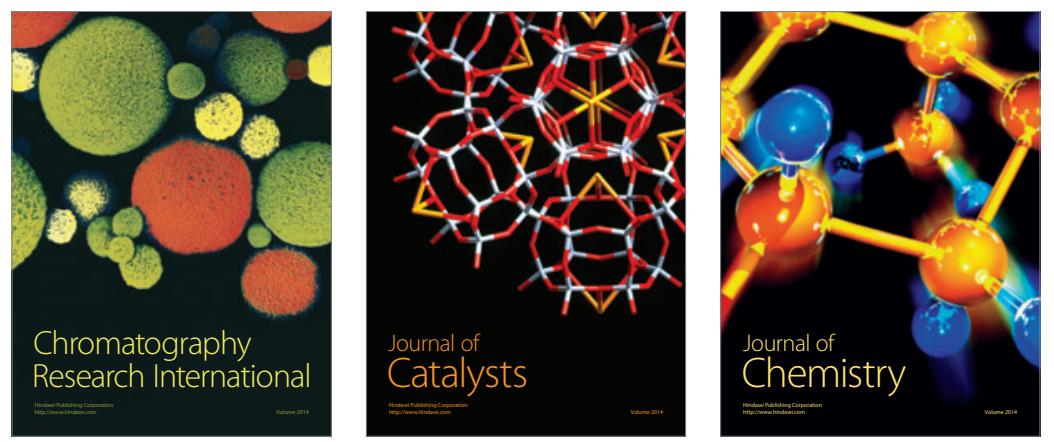
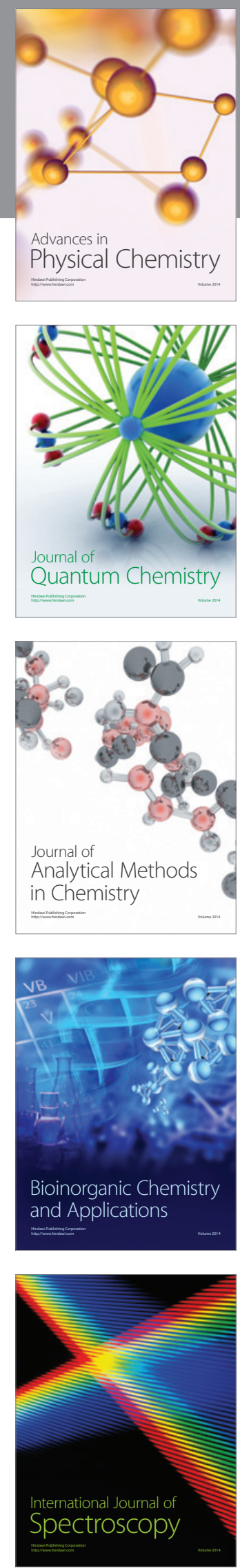